\title{
Reflets
}

Revue ontaroise d'intervention sociale et communautaire

\section{Mon corps, mon trésor}

\section{Jacqueline Plante}

Volume 6, numéro 1, printemps 2000

Approches d'intervention : définir et renouveler nos pratiques

URI : https://id.erudit.org/iderudit/026307ar

DOI : https://doi.org/10.7202/026307ar

Aller au sommaire du numéro

Éditeur(s)

Reflets : Revue ontaroise d'intervention sociale et communautaire

ISSN

1203-4576 (imprimé)

1712-8498 (numérique)

Découvrir la revue

Citer cet article

Plante, J. (2000). Mon corps, mon trésor. Reflets, 6(1), 226-229.

https://doi.org/10.7202/026307ar

Tous droits réservés (C) Reflets : Revue ontaroise d'intervention sociale et communautaire, 2000

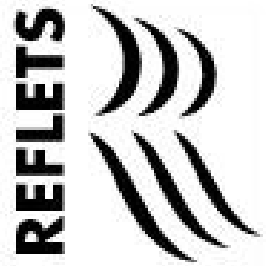

307ar

Ce document est protégé par la loi sur le droit d'auteur. L'utilisation des services d'Érudit (y compris la reproduction) est assujettie à sa politique d'utilisation que vous pouvez consulter en ligne.

https://apropos.erudit.org/fr/usagers/politique-dutilisation/ 


\title{
Mon corps, mon trésor
}

\author{
Jacqueline Plante, \\ infirmière hygiéniste, Unité sanitaire d'Algoma Sault Ste. Marie
}

En 1990, la petite ville de Prescott, située dans l'Est ontarien, était confrontée à la découverte d'un nombre important de situations d'agression sexuelle subies par les enfants de sa communauté. Sous la direction de l'ancienne mairesse Sandra Lawn, la communauté s'est mobilisée afin de répondre aux besoins des enfants victimes d'abus sexuel et discuter avec la communauté de leurs préoccupations face à ce problème. Cécile Loiselle, éducatrice en sexualité et Denise Gaulin, infirmière, toutes deux à l'emploi du Bureau de Santé publique du district de Leeds, Grenville et Lanark, ont alors mis à contribution leurs expertises en examinant les activités de prévention en fonction des facteurs suivants: l'efficacité au niveau de l'élimination de l'agression sexuelle, les groupes d'âges ciblés, la facilité d'adaptation d'une population d'enfants et d'adultes, l'impact pour les victimes, le message concernant la responsabilité de l'agresseur sexuel, la prévention de l'abus sexuel et la pertinence des activités proposées.

Dans la ville de Prescott, une enquête a renforcé les données d'études démontrant qu'on peut être victime à un très jeune âge d'abus sexuel et que celui-ci peut se poursuivre pendant de longues périodes avant d'être signalée. Dans la majorité des cas, l'enfant connait la personne qui l'a agressé sexuellement. À la lumière de ces faits, le Bureau de Santé publique se montre prudent face à l'utilisation des programmes, élaborés au cours de la dernière décennie, de prévention de l'abus sexuel. En lieu et place, il a créé un tout nouveau programme intitulé Care for Kids. Bien qu'il traite de la prévention de l'abus sexuel, ce programme porte principalement sur une éducation saine en matière de santé sexuelle, tout en tentant d'éviter les échecs et les limites d'autres programmes. 
À l'automne 1993, suite à un sondage, le personnel enseignant des écoles primaires anglophones de Sault Sainte-Marie identifie un besoin de formation en prévention des abus sexuels. Un comité formé de représentants des conseils scolaires, de la Société d'aide à l'enfance et de l'Unité sanitaire d'Algoma, se penche sur les programmes existants et se rend compte de leurs limites. La recherche de nouveau matériel mène à la découverte du programme Care for Kids.

L'Unité sanitaire d'Algoma et le Bureau de Santé publique de Leeds, Grenville et Lanark établissent donc un partenariat qui mène, en 1994, à la publication en anglais du programme dans un document illustré et intitulé Care for Kids.

Le programme cible les enfants âgés de trois à huit ans de la maternelle jusqu'à la troisième année. Il peut être utilisé par les enseignants, les parents, les grands-parents, les personnes offrant des soins aux enfants, les travailleurs des services à l'enfance, le personnel professionnel, les éducatrices et les éducateurs spécialisés. Son but est d'aider les adultes à discuter de la sexualité de façon positive avec les jeunes enfants, afin que ces derniers puissent se sentir à l'aise de parler d'abus sexuel, s'ils le désirent.

Care for Kids comporte sept thèmes abordés au moyen de messages, de chansons, d'activités de bricolage, etc. Ces thèmes comprennent les corps, les bébés, les sentiments, l'heure du coucher, les stéréotypes concernant les garçons et les filles, les touchers et les secrets. En plus du document, la trousse contient des poupées sexuées, sept livres, une cassette et une série de découpages.

En 1995, suite à la formation des intervenants, le programme Care for Kids s'implante progressivement dans les écoles séparées anglophones et dans les garderies. Les parents sont aussi invités à assister à des séances d'information pour connaître les objectifs véhiculés dans le programme et ainsi faire le lien avec l'école ou la garderie.

Entre temps, une coalition francophone qui voit le jour s'intéresse aussi à la question de la prévention des abus sexuels chez les enfants. Elle crée des partenariats qui regroupent au sein 
d'un comité consultatif, deux conseillères du Conseil scolaire catholique du Nouvel-Ontario, la directrice du Carrousel des amis, le Centre de ressources et l'infirmière hygiéniste francophone de l'Unité sanitaire d'Algoma. Pour atteindre notre objectif d'offrir le programme Care for Kids à la communauté francophone de Sault Sainte-Marie et du district d'Algoma, il faut aller chercher des fonds, rédiger des demandes à cet effet auprès des différents ministères, sensibiliser la communauté (comités de parents, clubs sociaux, Centre francophone, les associations et les comités voués à la prévention des abus sexuels), en plus de trouver un organisme pour nous parrainer. Le Centre francophone de Sault SainteMarie accepte de parrainer notre projet et nous recevons l'appui financier des ministères de la Santé, du Développement du Nord et des mines et des Affaires francophones, de l'Unité sanitaire d'Algoma, du Conseil scolaire catholique, des A.P.E. et des clubs sociaux.

La traduction du document qui doit aussi comporter l'adaptation culturelle du matériel, est confiée à l'auteure Cécile Loiselle, elle-même francophone. Par la suite, le comité consultatif procèdra à sa révision.

Après plusieurs années d'efforts et de ténacité, c'est en mars 1998 que le comité consultatif francophone réalise enfin ses objectifs, soit la traduction, l'adaptation culturelle et la mise en page du programme Care for Kids en français. Le magasin de jouets éducatifs Constellations se charge de monter les trousses, prépare les bons de commandes et assure la vente et la distribution pour les clientèles francophone et anglophone. Des invitations sont lancées aux enseignants, travailleurs des services à l'enfance et aux professionnels de la santé francophones de Sault Sainte-Marie et du district d'Algoma pour participer au premier atelier de formation du programme, animé par Cécile Loiselle et d'autres membres du comité. Une vingtaine de personnes s'inscrit à l'atelier. Une soirée d'information à l'intention des parents est aussi offerte. L'expérience fut un franc succès.

Depuis septembre 1998, les élèves de la maternelle des écoles élémentaires de Sault Sainte-Marie bénéficient du programme. De plus, le Conseil scolaire catholique du Nouvel-Ontario, dont 
le siège social est situé à Sudbury, a financé deux ateliers destinés au personnel enseignant de Sudbury et du district d'Algoma. Dernièrement, le comité francophone, avec l'accord des auteurs, a adopté Mon Corps, Mon Trésor comme appellation française du programme Care for Kids.

Les parents jouent un rôle primordial dans l'éducation sexuelle de leurs enfants, c'est donc à eux que revient en premier lieu la tâche importante d'aider leurs enfants dans leur épanouissement sexuel. Parfois les jeunes enfants comprennent ou interprètent mal le matériel qu'ils voient pour la première fois. Une mauvaise compréhension, particulièrement quand il s'agit de sexualité, peut parfois soulever les inquiétudes des parents et de la communauté, d'où l'importance d'offrir des sessions de formation aux parents et à des personnes de la communauté avant que le programme ne soit présenté aux enfants. Ces sessions peuvent aussi servir à l'éducation des adultes en matière de développement sexuel normal chez l'enfant et offrir des ressources pour faciliter l'éducation sur la sexualité dans la famille et la communauté.

En conclusion, les témoignages des personnes ayant participé au programme Care for Kids ou Mon Corps, Mon Trésor ont permis de constater qu'un sujet troublant, comme l'agression sexuelle des enfants, peut être abordé d'une manière ouverte, positive et saine. Les adultes disent se sentir plus à l'aise pour parler de sexualité avec leurs jeunes enfants. Quant à ceux-ci, ils acquerront une meilleure estime d'eux-mêmes, feront l'apprentissage d'un vocabulaire approprié sur la sexualité et auront une communication plus ouverte avec leurs parents et avec d'autres adultes. 